\title{
Long-term results of treatment of advanced dermatofibrosarcoma protuberans (DFSP) with imatinib mesylate - The impact of fibrosarcomatous transformation
}

\author{
P. Rutkowski ${ }^{\text {a, }}$, A. Klimczak ${ }^{\text {a }}$, I. Ługowska ${ }^{\text {a }}$, B. Jagielska ${ }^{\text {b }}$, \\ M. Wągrodzki ${ }^{\text {c }}$, M. Dębiec-Rychter ${ }^{d}$, B. Pieńkowska-Grela ${ }^{c}$, \\ T. Świtaj ${ }^{a}$ \\ a Department of Soft Tissue/Bone Sarcoma and Melanoma, Maria Sklodowska-Curie Memorial Cancer Center, \\ Institute of Oncology, Warsaw Poland \\ ${ }^{\mathrm{b}}$ Department of Oncology, Cardiooncology and Internal Medicine, Maria Sklodowska-Curie Memorial Cancer \\ Center, Institute of Oncology, Warsaw Poland \\ ${ }^{\mathrm{c}}$ Department of Pathology, Maria Sklodowska-Curie Memorial Cancer Center, Institute of Oncology, \\ Warsaw Poland \\ ${ }^{\mathrm{d}}$ Center for Human Genetics, KU, Leuven, Belgium
}

Accepted 14 March 2017

Available online

\begin{abstract}
Background: Dermatofibrosarcoma protuberans (DFSP) is rare, infiltrating dermal neoplasm, characterized by indolent growth and low probability of metastases. The first effective systemic therapy in DFSP introduced into clinical practice was imatinib, demonstrating high activity in advanced cases. The aim of the study was to perform an analysis of patients with advanced DFSP treated with imatinib, with or without surgery, in routine clinical practice with long-term follow-up.

Patients and methods: We analyzed the data of 31 Caucasian patients (14 male, 17 female; median age 56 years) with locally advanced/ initially inoperable and/or metastatic DFSP who started therapy with imatinib at initial dose $800 \mathrm{mg}$ daily between 12/2004 and 07/2014. All diagnoses were confirmed cytogenetically for the presence of specific COL1A1-PDGFB fusion. Median follow-up time was 5.3 years. Results: Metastases were present in 15 cases ( 8 - lungs, 5 - soft tissue, 2 - lymph nodes). Fibrosarcomatous transformation (FS-DFSP) was confirmed in 16 patients (52\%). 5-year progression-free survival (PFS) rate was 58\% (median 6.8 years), 5-year overall survival (OS) rate was $64 \%$ (median time for OS was not reached). The shorter PFS and OS correlated with FS-DFSP and presence of metastatic disease. 5-year PFS rate was $93 \%$ for classic DFSP and 33\% for FS-DFSP. The best overall responses were: 21 partial responses (68\%, including 8 FS-DFSP, but the responses were shorter than for classic DFSP), 6 stable disease (19\%) and 4 progressive diseases (13\%). Thirteen patients $(47 \%)$ underwent resection of residual disease and nine of them remained free of disease, although imatinib was discontinued. Median survival after progression on imatinib was 19 months, and longer survival were observed only in cases were rescue surgery/radiotherapy was possible.

Conclusions: Our results indicate the long-term activity of imatinib in therapy of inoperable and/or metastatic cases of DFSP, including FSDFSP. Some DFSP patients initially evaluated as unresectable/metastatic or necessitating mutilating surgery turned resectable after imatinib therapy and this rational approach leading to complete remission maybe potentially curative.
\end{abstract}

(C) 2017 Elsevier Ltd, BASO The Association for Cancer Surgery, and the European Society of Surgical Oncology. All rights reserved.

Keywords: Dermatofibrosarcoma protuberans; Fibrosarcomatous transformation; Imatinib

\footnotetext{
* Corresponding author. Department of Soft Tissue/Bone Sarcoma and Melanoma, Maria Sklodowska-Curie Memorial Cancer Center, Institute of Oncology, Roentgena 5, 02-781 Warsaw, Poland. Fax: +48 226439791.

E-mail address: piotr.rutkowski@coi.pl (P. Rutkowski).
} 


\section{Introduction}

Dermatofibrosarcoma protuberans (DFSP) is a rare soft tissue tumor, comprising approximately $1 \%$ of sarcomas, with typically indolent growth over years and probability of regional/distant metastases less than $5 \%$, especially in DFSP with fibrosarcomatous transformation (DFSPFS). ${ }^{1-8}$ The standard treatment of localized primary or recurrent cases is radical, wide local excision with clear margins, which may require reconstructive techniques and may cause functional and cosmetic disfigurement. $2,9,10$

From molecular point of view DFSP is typically characterized by a specific rearrangement of chromosomes 17 and 22 in the form of translocation $\mathrm{t}(17 ; 22)$ (q22; q13) that leads to the fusion of collagen type I A1-chain gene (COL1A1) to the platelet-derived growth factor B-chain gene $(P D G F B)$ - often visible in supernumerary ring chromosome. The result of this rearrangement is upregulation of the COL1A1-PDGFB fusion protein, processing to a mature homodimer PDGF-BB, and consequently continuous autocrine activation of the PDGFB receptor (PDGFRB), a protein tyrosine kinase acting as a potent growth factor. ${ }^{2,11-13}$ These mechanisms contribute directly to development and growth of DFSP, but also of giant cell fibroblastoma (GCF), which from a pathogenetic point of view can be called the juvenile form of DFSP. ${ }^{14}$

Advances in the understanding of the molecular mechanisms of DFSP have resulted in the introduction to clinical practice targeted therapy acting on PDGFRB. The first effective systemic therapy in DFSP introduced into clinical practice was imatinib mesylate..$^{2,15-18}$

Due to the rarity of the disease and the lack of ongoing prospective trials we have decided to assess therapeutic activity of imatinib in the largest presented group of patients with locally advanced and/or metastatic DFSP expressing COL1A/PDGFB fusion treated in one cancer center outside clinical trials with long-term follow-up. The aim of the study was to analyze distant outcomes (survival and recurrences) of imatinib-treated advanced DFSP and scopes of neoadjuvant strategies in routine practice.

\section{Patients and methods}

We analyzed all consecutive patients with locally advanced/metastatic DFSP treated with imatinib mesylate, from December 2004 to July 2014. Patients were treated at one reference center for treatment of soft tissue sarcomas. All patients provided a written informed consent to the treatment with imatinib. Approval by Institutional Review Board was also required.

All patients met the following criteria for imatinib therapy:

1) histological diagnosis of DFSP;

2) cytogenetic confirmation of presence of COL1A1/ PDGFB (by fluorescence in situ hybridization - FISH);
3) metastatic and/or locally inoperable lesions or advanced tumor possible only to remove with significant functional/cosmetic consequences (as assessed by multidisciplinary team)

4) measurable disease with photographic imaging of superficial lesions and or CT scans in case of visceral metastases;

5) WHO performance status $\leq 3$;

$6)$ adequate renal and liver function.

Adverse events during therapy were evaluated by Common Terminology Criteria for Adverse Events (CTCAE), version 4.0.

Objective responses were evaluated by serial measurements with photographic documentation of superficial lesions and contrast enhanced spiral CT in case of deep structures involvement according to RECIST 1.1. ${ }^{19}$ Possibility of resection of residual disease after response to therapy was assessed during every evaluation by two oncological surgeons. In case of such resection, as well as if postsurgical pathological evaluation confirmed microscopically, radical resection therapy with imatinib was stopped.

Progression-free survival (PFS) time was calculated from the date of the start of imatinib treatment to the date of the most recent follow-up, or progression or death due to the disease. Overall survival (OS) time was calculated from the date of the start of imatinib treatment to the date of the most recent follow-up or death due to the disease. Median follow-up time from the start of imatinib therapy was 5.3 years. The Kaplan-Meier method was used for analysis of survival curves, with the log-rank test for univariate comparison of the survival between groups. Differences were considered statistically significant if $\mathrm{p}$ values were $<0.05$. These statistical computations were performed using Statistica 7.1 software [Statsoft ${ }^{\circledR}$; Tulsa, $\mathrm{OK}]$.

\section{Results}

We retrospectively identified 31 patients treated with imatinib in initial dose $400 \mathrm{mg}$ bid, except one patient who started therapy from $400 \mathrm{mg}$ daily. Table 1 summarizes patient characteristics and clinical findings. Metastases were present in 15 cases (eight - lungs, five - soft tissue, two - lymph nodes). Fibrosarcomatous transformation (FSDFSP) was confirmed in 16 patients (52\%) and metastases occurred only in FS-DFSP cases.

In terms of efficacy overall clinical benefit of imatinib therapy (counted as the sum of complete remissions $\mathrm{CR}$, partial remissions $-\mathrm{PR}$ and stable disease $-\mathrm{SD}$ rates) was $87 \%$ (Table 2). The best overall responses were: 21 partial responses $(68 \%$, including eight FS-DFSP, but the responses were shorter than for classic DFSP), six stable disease (19\%) and four progressive diseases (13\%). 
Table 1

Patient characteristics and clinical findings.

\begin{tabular}{ll}
\hline & $\mathrm{N}=31(\%)$ \\
\hline Gender & \\
Male & $14(45 \%)$ \\
Female & $17(55 \%)$ \\
Age - median (range) [years] & $56(20-71)$ \\
Primary tumor location & \\
$\quad$ Trunk & $17(55 \%)$ \\
Head & $7(22.5 \%)$ \\
Extremities & $7(22.5 \%)$ \\
Primary tumor & $7(23 \%)$ \\
Recurrent tumor & $9(29 \%)$ \\
Metastatic & $15(48 \%)$ \\
Classic DFSP & $15(48 \%)$ \\
Fibrosarcomatous DFSP & $16(52 \%)$ \\
Location of metastases & \\
Lungs & $8(54 \%)$ \\
Soft tissues & $5(33 \%)$ \\
Lymph nodes & $2(13 \%)$ \\
\hline
\end{tabular}

5-year progression-free survival (PFS) rate was 58\% (median 6.8 years), 5-year overall survival (OS) rate was 64\% (median time for OS was not reached) [Fig. 1A and $B]$. The shorter PFS and OS correlated with FS-DFSP $(\mathrm{p}<0.01)$ and presence of metastatic disease $(\mathrm{p}<0.05)$ [Fig. 2A and B]. 5-year PFS rate was 93\% for classic DFSP and 33\% for FS-DFSP $(\mathrm{p}<0.01)$.

Thirteen patients $(47 \%)$ underwent resection of localized residual disease (after median time of 4 months of preoperative imatinib, 1 was FS-DFSP) and nine of them remained free of disease, although imatinib was discontinued. Minimal macroscopic assessed margins were $1 \mathrm{~cm}$ (measured from the residual tumor after imatinib therapy) and contained the tattoo points (if marked) of initial tumor. Postoperative assessment revealed macroscopically negative margins (R0) in 8 cases, three of five patients with microscopically non-radical resection (R1) continued imatinib therapy postoperatively. Disease relapsed in 3 patients after R1 resection (two of them continued imatinib

Table 2

The clinical efficacy of imatinib, progression and survival status for entire group of patients.

\begin{tabular}{ll}
\hline & Total $(\mathrm{N}=31)$ \\
\cline { 2 - 2 } & $\mathrm{N}(\%)$ \\
\hline Progression status & \\
$\quad$ Progression free & $19(61)$ \\
$\quad$ Progression & $12(39)$ \\
Survival status & \\
Alive & $22(71)$ \\
Dead & $9(29)$ \\
Best overall response & \\
Partial response & $21(68)$ \\
[including FS-DFSP] & 8 of 16 \\
Stable disease & $6(19)$ \\
Progressive disease & $4(13)$ \\
\hline
\end{tabular}

postoperatively and recurrence was re-resected in 2 cases) and in 1 patient in R0 group. Additionally two metastatic FS-DFSP patient underwent metastasectomy and continued imatinib postoperatively.Median survival after progression on imatinib was 19 months, and longer survival were observed only in cases were rescue surgery/radiotherapy was possible.

Adverse events were relatively common during imatinib therapy, but no new toxicities were reported (Table 3). Eleven patients $(35 \%)$ treated with initial imatinib dose $400 \mathrm{mg}$ bid had finally reduction of the dose to 400-600 mg daily due to adverse events as recommended by manufacturer or required by patient.

\section{Discussion}

In this study we have presented the largest one institution series of advanced DFSP patients treated with imatinib with the longest follow-up. The results of this study confirmed that majority of advanced DFSP patients benefit from imatinib therapy with median PFS exceeding 5 years.

Taking into account all limitations related to retrospective analysis, these results seem to be comparable to outcomes of reported small prospective trials, but also they provide new information on the activity of imatinib in daily clinical practice, especially in the subgroup of FS-DFSP patients with more aggressive course of disease. Moreover, our series comprise unique homogenous group of patients with confirmed cytogenetically presence of COL1A1$P D G F B$ fusion gene, which is especially important as a target of imatinib therapy in FS-DFSP.

Although rarely DFSP is present as inoperable or metastatic, imatinib mesylate has become a gold standard treatment in such cases. ${ }^{2,20,21}$ Until now reports of several cases and small phase II trials demonstrated the usefulness of imatinib in metastatic and localized unresectable DFSP. ${ }^{15-18,22-25}$ We have confirmed that FS-DFSP still have sensitivity to imatinib, although responses last shorter $^{26}$ with median PFS approximately 1.5 years in our series of patients. Moreover, metastatic disease is closely related to fibrosarcomatous transformation. ${ }^{27,28}$

Majority of patients treated with imatinib experience adverse events during treatment, but almost all toxic effects are mild and manageable. There are no disease-specific side effects and these are similar to those observed in patients with gastrointestinal stromal tumor (GIST). Although imatinib is well tolerated but if used in initial dose of $800 \mathrm{mg}$ daily, the dose reduction is required in significant proportion of patients (35\% in our series).

The preoperative imatinib strategy for tumor downstaging and decreasing of excision morbidity by tissue-sparing leading to diminish cosmetic disfigurement or functional impairment appears very attractive. Kerob et al. ${ }^{29}$ presented report on 25 resectable DFSP (median size $-4.5 \mathrm{~cm}$ ) treated in phase II trial with neoadjuvant imatinib at the dose of $600 \mathrm{mg}$ daily for two months, objective partial 

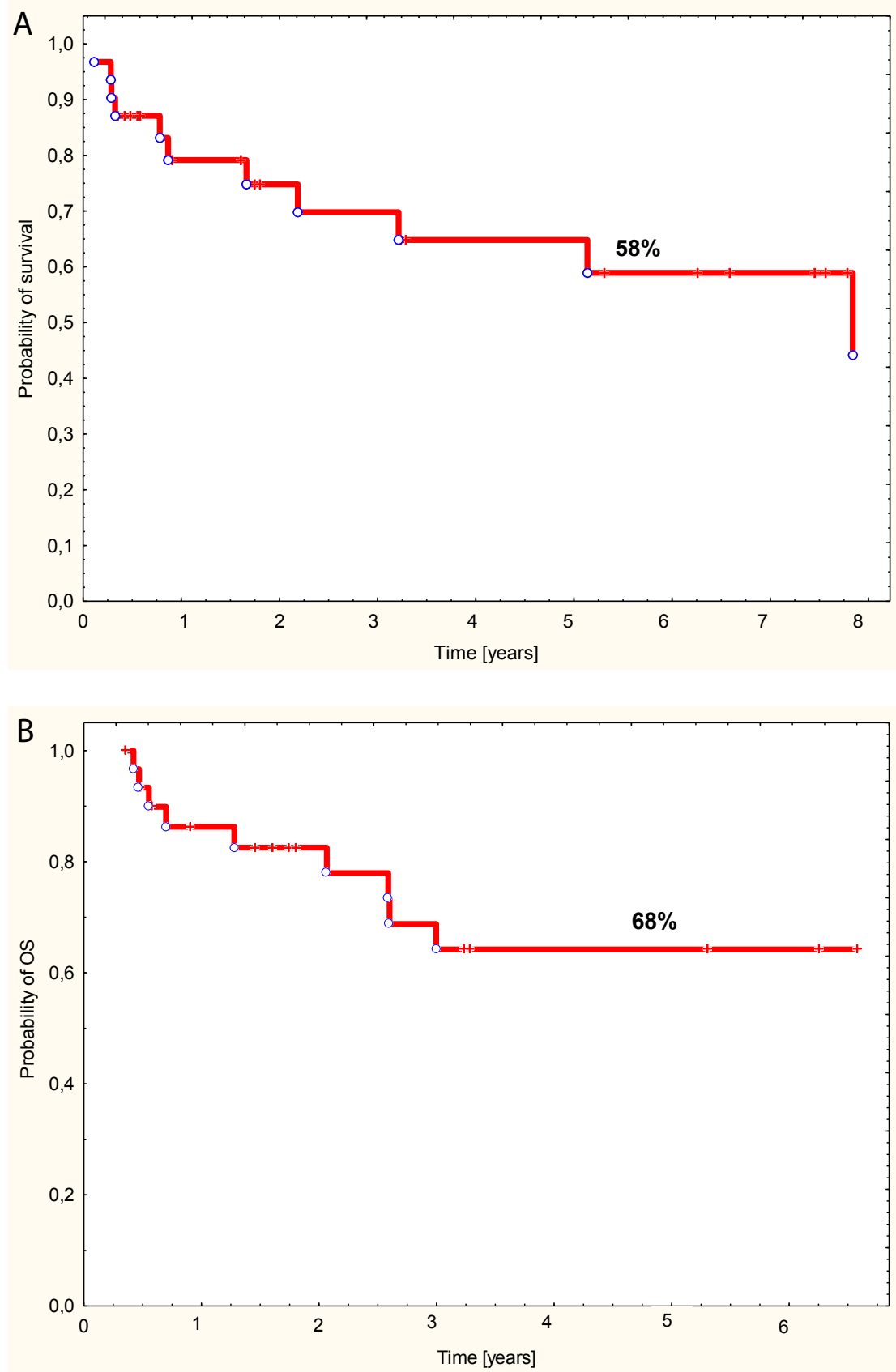

Figure 1. A. Progression-free survival from date of imatinib start. B. Overall survival from date of imatinib start.

response according to RECIST was observed in only 9 cases $(36 \%)$. It is likely, that the response rate in this trial would have been significantly higher if the preoperative treatment duration would have been prolonged, as it was in our cases. Ugurel et al. ${ }^{30}$ presented the results of multicenter phase II trial on neoadjuvant imatinib therapy at dose of $600 \mathrm{mg}$ daily in locally advanced primary or recurrent DFSP with clinical benefit exceeding 90\%. The definitive surgical resection of the tumor was performed in 13 of 14 patients.

In our 13 of 16 non-metastatic, locally advanced patients underwent resection of residual disease after partial response to imatinib therapy and nine of them had no disease recurrence during further follow-up. Current results indicate that some DFSP patient initially evaluated as unresectable/metastatic or necessitating mutilating surgery 

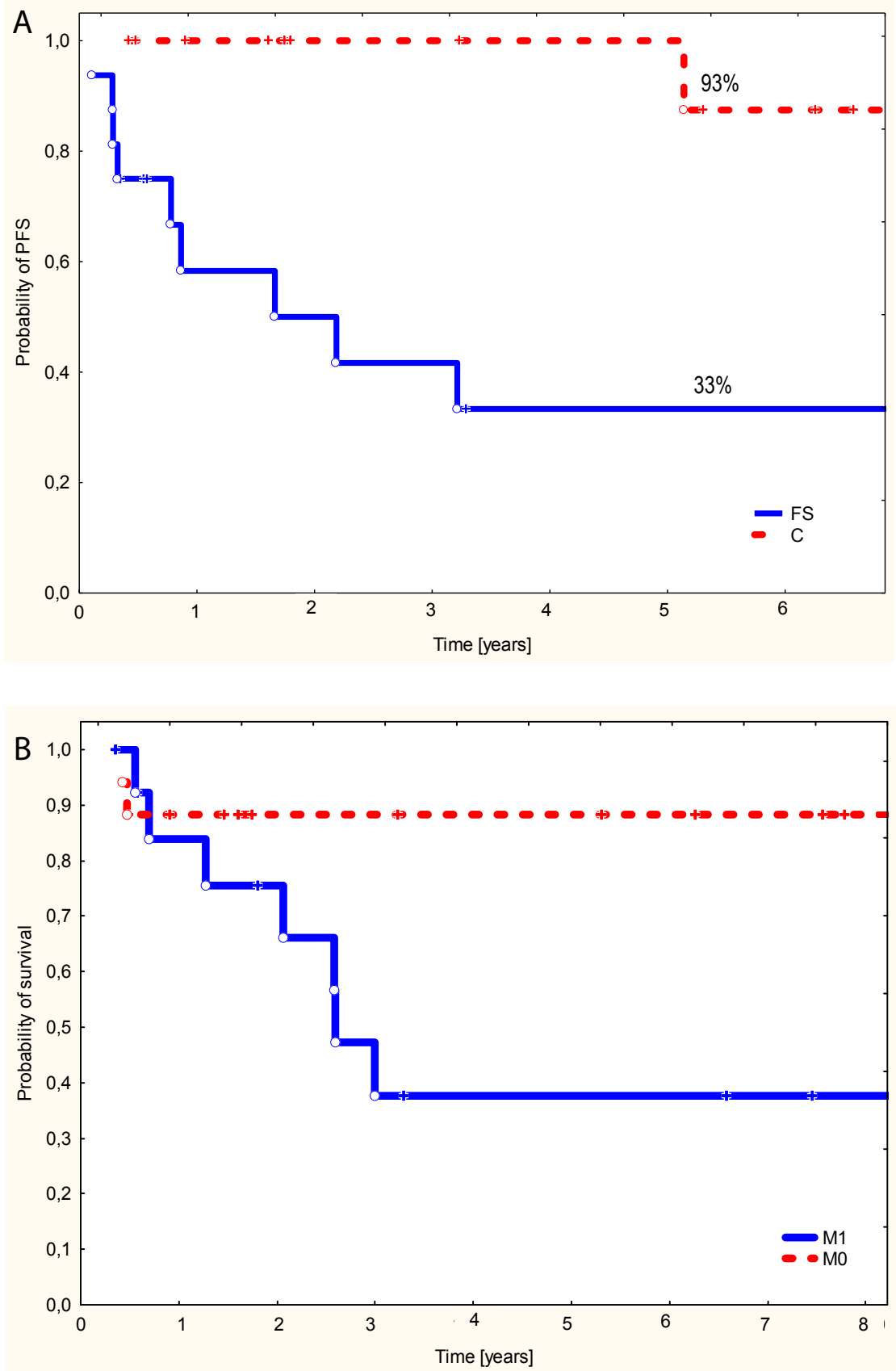

Figure 2. A. Progression-free survival according to fibrosarcomatous transformation $(\mathrm{p}<0.01)$. B. Overall survival according to presence of metastases $(\mathrm{p}<0.05)$.

turned resectable after imatinib therapy. This rational approach leading to complete remission maybe potentially curative (Fig. 3). ${ }^{31-34}$ Further studies are necessary for elucidating whether preoperative imatinib therapy reduces the need for wide surgical margins or whether imatinib has activity as adjuvant therapy in cases of positive margins after excision (if re-excision is not feasible) or in other high-risk patients (e.g. FS-DFSP or located near critical anatomical sites).

It is little know about mechanism of resistance to imatinib. According to Ugurel et al. a weak PDGFRB phosphorylation seems to be associated with non-response to imatinib. ${ }^{30}$ According to Eilers CDKN2A/p16 loss implicates $\mathrm{CDK} 4$ as a possible target in imatinib-resistant 
Table 3

Adverse events during imatinib therapy in DFSP.

\begin{tabular}{ll}
\hline Adverse event and grade CTCAE & $\mathrm{N}(\%)$ \\
\hline Edema/fluid retention & $24(77 \%)$ \\
G1 & $3(10 \%)$ \\
G2 & $1(3 \%)$ \\
G3 & \\
Fatigue & $6(20 \%)$ \\
G1 & $1(3 \%)$ \\
G2 & $1(3 \%)$ \\
G3 & \\
Skin toxicity & $6(20 \%)$ \\
G1 & $2(6 \%)$ \\
G2 & \\
Nausea & $5(16 \%)$ \\
G1 & $2(6 \%)$ \\
G2 & \\
Diarrhea & $4(13 \%)$ \\
G1 & $2(6 \%)$ \\
G2 & $1(3 \%)$ \\
G3 & \\
Anemia & $8(25 \%)$ \\
G1 & $3(9.7 \%)$ \\
G2 & \\
Neutropenia & $3(9.7 \%)$ \\
G3 & \\
Thrombocytopenia & $3(9.7 \%)$ \\
G1 & $1(3 \%)$ \\
G2 & $2(6.4 \%)$ \\
\hline G3 &
\end{tabular}

DFSP. ${ }^{35}$ Tazzari et al. imply the future utility of immunotherapy in advanced DFSP. ${ }^{36}$

In series from Memorial Sloan-Kettering Cancer Cen$\operatorname{ter}^{37}$ two patients were treated with sorafenib after failure on imatinib and one partial response was observed. Similar report was provided by Kamar et al., ${ }^{38}$ where locally invasive DFSP after failure of radiation therapy and imatinib $(400-800 \mathrm{mg})$ was successfully treated with sorafenib $800 \mathrm{mg}$ daily for 5 months. The single patient who developed secondary resistance to imatinib in German phase II trial responded transiently to second-line therapy with sunitinib, which may be explained by the binding capacity of sunitinib to PDGFRB, which is about 10 times more than that of imatinib. ${ }^{30}$ Chinese study on 30 imatinib-resistant metastatic DFSP suggest also the efficacy of sunitinib in second-line therapy with median PFS and OS -19 and 27 months, respectively. ${ }^{39}$

In conclusion, our results with median follow-up exceeding 5 years indicate the long-term activity of imatinib in therapy of inoperable and/or metastatic cases of DFSP harboring COL1A1-PDGFB fusion, ${ }^{40-42}$ with an objective response rate exceeding $50 \%$, and that imatinib is also active in aggressive fibrosarcomatous DFSP. ${ }^{28,43}$ The activity of imatinib is limited in time, especially in FS-DFSP, and still no effective, proven options after progression exist. Some DFSP patient initially evaluated as un-resectable/ metastatic or demanding mutilating surgery turned resectable after imatinib therapy and this rational approach leading to complete remission is potentially curative.
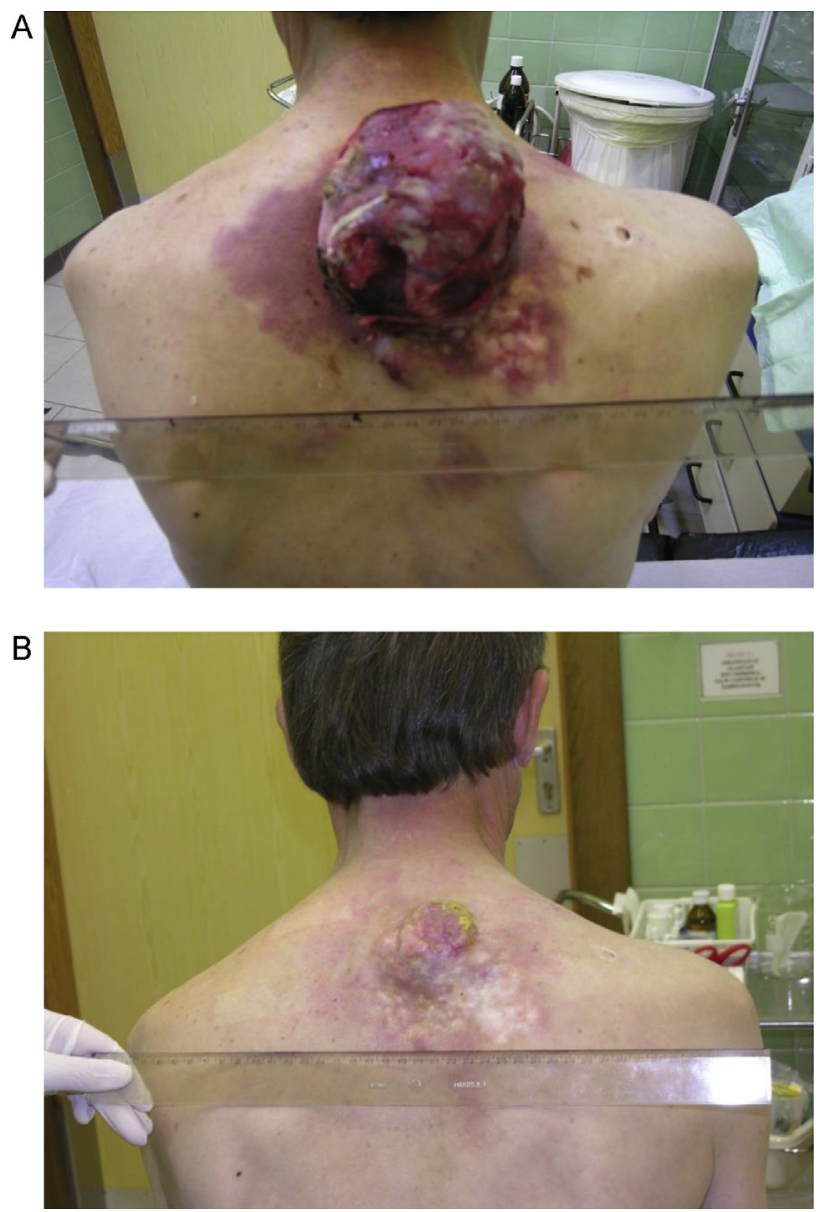

Figure 3. Photographs demonstrating the response of advanced multifocal DFSP on the back to imatinib (A. before therapy, B. after 3 months of imatinib therapy that was followed by microscopically radical resection of the tumor and no recurrent disease during 5 years of follow-up).

Current therapy of DFSP with $t(17 ; 22)$ translocation should be definitively conducted by multidisciplinary team, including oncological surgeon, to consider the use of imatinib mesylate as initial therapy to decrease possible extent of surgery and related morbidity.

\section{Conflict of interest}

Piotr Rutkowski hasreceived honoraria and was a member of Advisory Board for Novartis.

\section{Funding source}

No external sponsors were involved into this study.

\section{References}

1. Lemm D, Mugge LO, Mentzel T, et al. Current treatment options in dermatofibrosarcoma protuberans. J Cancer Res Clin Oncol 2009; 135:653-65. 
2. Rutkowski P, Debiec-Rychter M. Current treatment options for dermatofibrosarcoma protuberans. Expert Rev Anticancer Ther 2015;15(8): 901-9.

3. Criscito MC, Martires KJ, Stein JA. Prognostic factors, treatment, and survival in dermatofibrosarcoma protuberans. JAMA Dermatol 2016 Dec 1;152(12):1365-71.

4. Kreicher KL, Kurlander DE, Gittleman HR, Barnholtz-Sloan JS, Bordeaux JS. Incidence and survival of primary dermatofibrosarcoma protuberans in the United States. Dermatol Surg 2016 Jan;42(Suppl 1): S24-31.

5. Liang CA, Jambusaria-Pahlajani A, Karia PS, et al. A systematic review of outcome data for dermatofibrosarcoma protuberans with and without fibrosarcomatous change. J Am Acad Dermatol 2014;71: 781-6.

6. Mentzel T, Beham A, Katenkamp D, et al. Fibrosarcomatous 'highgrade' dermatofibrosarcoma protuberans: clinicopathological and immunohistochemical study of 41 cases with emphasis on prognostic significance. Am J Surg Pathol 1998;22:576-87.

7. Stacchiotti S, Pedeutour F, Negri T, et al. Dermatofibrosarcoma protuberans-derived fibrosarcoma: clinical history, biological profile and sensitivity to imatinib. Int J Cancer 2011;129(7):1761-7.

8. Kuzel P, Mahmood MN, Metelitsa AI, Salopek TG. A clinicopathologic review of a case series of dermatofibrosarcoma protuberans with fibrosarcomatous differentiation. J Cutan Med Surg 2015 Jan-Feb;19(1):28-34.

9. Fiore M, Miceli R, Mussi C, et al. Dermatofibrosarcoma protuberans treated at a single institution: a surgical disease with a high cure rate. J Clin Oncol 2005;23:7669-75.

10. Foroozan M, Sei JF, Amini M, et al. Efficacy of Mohs micrographic surgery for the treatment of dermatofibrosarcoma protuberans: systematic review. Arch Dermatol 2012;148:1055-63.

11. Sandberg AA, Bridge JA. Updates on the cytogenetics and molecular genetics of bone and soft tissue tumors: dermatofibrosarcoma protuberans and giant cell fibroblastoma. Cancer Genet Cytogenet 2003; 140:1-12.

12. Sirvent N, Maire G, Pedeutour F. Genetics of dermatofibrosarcoma protuberans family of tumors: from ring chromosomes to tyrosine kinase inhibitor treatment. Genes Chromosom Cancer 2003;37(1): $1-19$.

13. Simon MP, Pedeutour F, Sirvent N, et al. Deregulation of the platelet derived growth factor B-chain gene via fusion with collagen gene COL1A1 in dermatofibrosarcoma protuberans and giant-cell fibroblastoma. Nat Gen 1997;15:95-8.

14. Terrier-Lacombe MJ, Guillou L, Maire G, et al. Dermatofibrosarcoma protuberans, giant cell fibroblastoma, and hybrid lesions in children: clinicopathologic comparative analysis of 28 cases with molecular data-a study from the French Federation of Cancer Centers Sarcoma Group. Am J Surg Pathol 2003;27(1):27-39.

15. McArthur GA, Demetri GD, van Oosterom AT, et al. Molecular and clinical analysis of locally advanced dermatofibrosarcoma protuberans treated with imatinib: imatinib Target Exploration Consortium Study B2225. J Clin Oncol 2005;23:866-73.

16. Heinrich MC, Joensuu H, Demetri GD, et al. Phase II, open-label study evaluating the activity of imatinib in treating life-threatening malignancies known to be associated with imatinib-sensitive tyrosine kinases. Clin Cancer Res 2008;14:2717-25.

17. Rutkowski P, Van Glabbeke M, Rankin CJ, et al. Imatinib mesylate in advanced dermatofibrosarcoma protuberans (DFSP): pooled analysis of two phase II clinical trials. J Clin Oncol 2010;28:1772-9.

18. Rutkowski P, Dębiec-Rychter M, Nowecki ZI, et al. Treatment of advanced dermatofibrosarcoma protuberans with imatinib mesylate with or without surgical resection. J Eur Acad Dermatol Venereol 2011;25(3):264-70.

19. Eisenhauer EA, Therasse P, Bogaerts J, et al. New response evaluation criteria in solid tumours: revised RECIST guideline (version 1.1). Eur $J$ Cancer 2009;45(2):228-47.

20. NCCN Guidelines v. 1. Dermatofibrosarcoma protuberans 2016.
21. Saiag P, Grob JJ, Lebbe C, et al. Diagnosis and treatment of dermatofibrosarcoma protuberans. European consensus-based interdisciplinary guideline. Eur J Cancer 2015;51(17):2604-8.

22. Labropoulos SV, Fletcher JA, Oliveira AM, et al. Sustained complete remission of metastatic dermatofibrosarcoma protuberans with imatinib mesylate. Anticancer Drugs 2005;16:461-6.

23. Mizutani K, Tamada Y, Hara K, et al. Imatinib mesylate inhibits the growth of metastatic lung lesions in a patient with dermatofibrosarcoma protuberans. Br J Dermatol 2004;151:235-7.

24. Wang C, Luo Z, Chen J, et al. Target therapy of unresectable or metastatic dermatofibrosarcoma protuberans with imatinib mesylate: an analysis on 22 Chinese patients. Medicine 2015 May;94(17):e773. (Baltimore).

25. Tatai T, Gomi D, Fukushima T, et al. Effectiveness of imatinib mesylate treatment in a patient with dermatofibrosarcoma protuberans with pulmonary and pancreatic metastases. Intern Med 2016;55(17):250711.

26. Gronchi A, Stacchiotti S, Pedeutour F, et al. Response to imatinib mesylate (IM) in fibrosarcoma (FS) arising in dermatofibrosarcoma protuberans (DFSP). J Clin Oncol May 2008;26(15_suppl). 10593-10593.

27. Stacchiotti S, Pantaleo MA, Negri T, et al. Efficacy and biological activity of imatinib in metastatic dermatofibrosarcoma protuberans (DFSP). Clin Cancer Res 2016 Feb 15;22(4):837-46.

28. Hayakawa K, Matsumoto S, Ae K, et al. Risk factors for distant metastasis of dermatofibrosarcoma protuberans. J Orthop Traumatol 2016 Sep;17(3):261-6.

29. Kérob D, Porcher R, Vérola O, et al. Imatinib mesylate as a preoperative therapy in dermatofibrosarcoma: results of a multicenter phase II study on 25 patients. Clin Cancer Res 2010;16:3288-95.

30. Ugurel S, Mentzel T, Utikal J, et al. Neoadjuvant imatinib in advanced primary or locally recurrent dermatofibrosarcoma protuberans: a multicenter phase II DeCOG trial with long-term follow-up. Clin Cancer Res 2014;20(2):499-510.

31. Lemm D, Muegge LO, Hoeffken K, et al. Remission with Imatinib mesylate treatment in a patient with initially unresectable dermatofibrosarcoma protuberans-a case report. Oral Maxillofac Surg 2008; 12:209-13.

32. Han A, Chen EH, Niedt G, Sherman W, Ratner D. Neoadjuvant imatinib therapy for dermatofibrosarcoma protuberans. Arch Dermatol 2009;145:792-6.

33. Wicherts DA, Coevorden F, Klomp HM, et al. Complete resection of recurrent and initially unresectable dermatofibrosarcoma protuberans downsized by Imatinib. World J Surg Oncol 2013;11:59.

34. Johnson-Jahangir H, Sherman W, Ratner D. Using imatinib as neoadjuvant therapy in dermatofibrosarcoma protuberans: potential pluses and minuses. J Natl Compr Canc Netw 2010;8:881-5.

35. Eilers G, Czaplinski JT, Mayeda M, et al. CDKN2A/p16 loss implicates CDK4 as a therapeutic target in imatinib-resistant dermatofibrosarcoma protuberans. Mol Cancer Ther 2015;14(6):1346-53.

36. Tazzari M, Indio V, Vergani B, et al. Adaptive immunity in fibrosarcomatous dermatofibrosarcoma protuberans and response to imatinib treatment. J Investig. Dermatol 2017;137(2):484-93.

37. Fields RC, Hameed M, Qin L-X, et al. Dermatofibrosarcoma protuberans (DFSP): predictors of recurrence and the use of systemic therapy. Ann Surg Oncol 2011;18:328-36.

38. Kamar FG, Kirouz VF, Sabri AN. Dermatofibrosarcoma protuberans (DFSP) successfully treated with sorafenib: case report. Clin Sarcoma Res 2013;3:5.

39. Fu Y, Kang H, Zhao H, et al. Sunitinib for patients with locally advanced or distantly metastatic dermatofibrosarcoma protuberans but resistant to imatinib. Int J Clin Exp Med 2015;8(5):8288-94.

40. Kerob D, Pedeutour F, Leboeuf C, et al. Value of cytogenetic analysis in the treatment of dermatofibrosarcoma protuberans. J Clin Oncol 2008;26:1757-9.

41. Patel KU, Szabo SS, Hernandez VS, et al. Dermatofibrosarcoma protuberans COL1A1-PDGFB fusion is identified in virtually all 
dermatofibrosarcoma protuberans cases when investigated by newly developed multiplex reverse transcription polymerase chain reaction and fluorescence in situ hybridization assays. Hum Pathol 2008;39: 184-93.

42. Szollosi Z, Scholtz B, Egervari K, Nemes Z. Transformed dermatofibrosarcoma protuberans: real time polymerase chain reaction detection of COL1A1-PDGFB fusion transcripts in sarcomatous areas. J Clin Pathol 2007;60(2):190-4.

43. Llombart B, Serra-Guillén C, Monteagudo C, López Guerrero JA, Sanmartín O. Dermatofibrosarcoma protuberans: a comprehensive review and update on diagnosis and management. Semin Diagn Pathol 2013;30(1):13-28. 